\title{
ESTUDIO SOBRE LAS ESTRATEGIAS DE APRENDIZAJE Y EL AUTOCONCEPTO EN ALUMNOS DE ESO Y BACHILLERATO
}

\author{
Ángela Lope Álvarez \\ alope@unizar.es \\ José Luis Antoñanzas Laborda \\ Tania Carbonell \\ Universidad de Zaragoza
}

Fecha de Recepción: 20 Febrero 2018

Fecha de Admisión: 10 Abril 2018

\section{RESUMEN}

La planificación es un aspecto muy importante en el estudio de los alumnos, son las estrategias de aprendizaje un pilar fundamental en el desarrollo del estudio y su influencia en el rendimiento académico es crucial. Se investigó para conocer cómo eran las estrategias de aprendizaje y técnicas de estudio utilizadas por los estudiantes y además se administró un cuestionario para evaluar el autoconcepto de los estudiantes. Posteriormente se realizaron diferentes análisis estadísticos y se encontraron correlaciones estadísticamente significativas entre autoconcepto y estrategias de aprendizaje con nuestras variables sexo, curso y edad. lación

Palabras clave: planificación; estrategias aprendizaje; técnicas de estudio; autoconcepto; corre-

\section{ABSTRACT}

Study about learning strategies and self-concept in ESO and Bachillerato students.

Planning is a very important aspect in the study of students, learning strategies are a fundamental pillar in the development of the study and its influence on academic performance is crucial. It was researched to know how the learning strategies and study techniques used by the students were and also a questionnaire was administered to evaluate the self-concept of the students. Subsequently, different statistical analyzes were performed and statistically significant correlations were found between self-concept and learning strategies with our sex, course and age variables.

Keywords: planning; learning strategies; study techniques; self-concept; correlation

\section{ANTECEDENTES DE LA TEMÁTICA A TRATAR}

Uno de los grandes retos educativos de la educación actual es adaptarse a la diversidad existente en el aprendizaje de los alumnos, ya que se ha demostrado que no todos aprenden igual (Monereo, Pozo y Castello, 2001). 


\section{ESTUDIO SOBRE LAS ESTRATEGIAS DE APRENDIZAJE Y EL AUTOCONCEPTO EN ALUMNOS DE ESO Y BACHILLERATO}

Son las reformas educativas las que dan cuenta de ello además de que no podemos obviar la importancia creciente de las nuevas tecnologías en la enseñanza y su repercusión entre los jóvenes en su forma de aprender y relacionarse (Garaigordobil, Pérez, y Mozaz, 2008). Las estrategias de aprendizaje son por tanto uno de los temas que siguen estando a la orden del día en la investigación educativa.

Existen multitud de estudios que estudian como aprenden los alumnos, el uso de estrategias de aprendizaje 0 cómo se enseñan en el contexto escolar (Aguilar, 2010; Gargallo, Suárez, Ferreras, 2007; Herrera-Torres, Lorenzo-Quiles, 2009). Por otro lado, existen investigaciones que tratan sobre el autoconcepto o se centran en alguna de sus dimensiones (Amezcua y Pichardo, 2000; Costa y Tabernero, 2012; Infante, Morena, García, Sánchez, Hierrezuelo y Muñoz, 2002; Inglés, Pastor, Torregrosa, Redondo y García-Fernández, 2009), pero son pocos los trabajos que aglutinan el estudio de las estrategias de aprendizaje y el autoconcepto, que el presente estudio hace.

Por todo ello esta investigación pretende conocer las estrategias de aprendizaje y el autoconcepto de estudiantes de ESO y Bachillerato y las relaciones entre ellas con las variables sexo, edad y curso. Se utilizó la metodología cuantitativa con un diseño descriptivo simple y el uso de cuestionarios para llevarlo a cabo.

\section{OBJETIVOS DE LA INVESTIGACIÓN}

Esta investigación tenía un carácter exploratorio por lo que su objetivo primero era conocer más a fondo las estrategias de aprendizaje y el autoconcepto en nuestra muestra del que se desprenderán los objetivos específicos como son analizar las correlaciones existentes entre el autoconcepto y las estrategias de aprendizaje con las variables sexo, edad y curso.

\section{MÉTODO}

\section{Muestra}

Participaron en el estudio un total de 525 sujetos ( 271 hombres y 254 mujeres). Nuestra muestra estaba compuesta por alumnos de un instituto de Zaragoza desde primero de la ESO hasta segundo de Bachillerato. La edad de los sujetos estaba comprendida entre los 12 y los 19 años (M $=14,57 \pm 1,641)$.

\section{I \\ nstrumentos}

Para medir la variable de autoconcepto se utilizó el AFA (Autoconcepto Forma A) de (Musitu, García y Gutiérrez, 1994) compuesto por 36 ítems que evalúan el autoconcepto y sus diferentes vertientes: social, académica, emocional, familiar y física. Las alternativas de respuesta a cada ítem son de 1 a 3 en el que 1 se refiere a algo que ocurre "siempre", 2 se refiere a algo que ocurre "algunas veces" y 3 se refiere a algo que "nunca" sucede. El cuestionario mencionado tiene una consistencia interna 0 fiabilidad, evaluada a través de la prueba estadística Alfa de Cronbach, de 0.85 .

Para evaluar la variable de estrategias de aprendizaje se ha utilizado un cuestionario adaptado a nuestra investigación realizado por la Universidad de Granada compuesto por 32 ítems agrupados en diferentes bloques: datos sociodemográficos, lugar y condiciones de estudio, organización del estudio, estrategias de aprendizaje y por último satisfacción con las calificaciones obtenidas. Las alternativas de respuesta podían ser de diversa índole: dicotómicas (Si/No), relacionadas con la frecuencia (Nunca/A veces/A menudo/Siempre) o de respuesta abierta cuando se les preguntaba cuántas horas dedicaban semanalmente al estudio. El cuestionario mencionado tiene una consistencia interna o fiabilidad, evaluada a través de la prueba estadística Alfa de Cronbach, de 0.7. 


\section{Procedimiento}

Los alumnos fueron informados de la finalidad de la investigación y en horario lectivo, en la hora de tutoría, recibieron las instrucciones para contestar los cuestionarios, dadas por un miembro del equipo investigador.

\section{RESULTADOS}

Los datos de los 525 sujetos fueron codificados y analizados mediante el paquete estadístico SPSS 19.0. Se analizaron las relaciones entre variables mediante correlaciones de Pearson.

En primer lugar, se realizaron correlaciones utilizando las puntaciones en el AFA (Autoconcepto Forma A) y sus diferentes subescalas con las variables sexo, edad y curso, los resultados aparecen en la Tabla 1.

Autoconcepto y sexo: nos encontramos diferencias estadísticamente significativas $(p<, 01)$ en autoconcepto emocional $(-, 229)$ con una correlación baja y $(p<, 05)$ en autoconcepto familiar $(, 106)$ con una correlación baja.

Autoconcepto y edad: únicamente nos encontramos diferencias estadísticamente significativas $(p<, 01)$ en autoconcepto académico $(-, 177)$ con una correlación baja.

Autoconcepto y curso: únicamente nos encontramos diferencias estadísticamente significativas $(p<, 05)$ en autoconcepto académico $(-, 105)$ con una correlación baja.

Tabla 1. Correlaciones entre Autoconcepto y sexo, edad y curso

\begin{tabular}{llllll}
\hline & \multicolumn{4}{l}{ Autoconcepto Autoconcepto Autoconcepto } & \multicolumn{3}{l}{ Autoconcepto Autoconcepto } \\
& académico & social & emocional & familiar & Total \\
\hline Sexo &, 003 &,- 059 &,$- 229^{* *}$ &, $106^{*}$ &,$- 112^{*}$ \\
Edad &,$- 177^{* *}$ &,- 063 &, 066 &,- 088 &,- 097 \\
Curso &,$- 105^{*}$ &,- 005 &, 052 &,- 055 &,- 044 \\
\hline
\end{tabular}

$* * \mathrm{p}<.01 \quad * \mathrm{p}<.05$

En segundo lugar, se efectuaron correlaciones utilizando las puntuaciones en el cuestionario de Técnicas de estudio y estrategias de aprendizaje, con las variables sexo, edad y curso, los resultados aparecen en las Tabla 2 y Tabla 3.

Las estrategias de aprendizaje analizadas eran las siguientes:

Relación: "cuando estudio relaciono los contenidos de la materia con otras asignaturas"

Biblio: "amplio información con bibliografía complementaria

Materiales: "cuando estudio dispongo de toda la información y materiales necesarios"

Lectura: "antes de estudiar en profundidad suelo hacer una lectura superficial"

Subrayar: "subrayo el material de estudio"

Esquema: "hago esquemas del material a estudiar"

Palabra: "en la realización de esquemas utilizo muchas palabras"

Resumen: "realizo un resumen de los temas a estudiar"

Mapas: "hago mapas conceptuales de los temas de cada una de las materias para estudiar posteriormente"

Diccionario: "los temas que no entiendo nos consulto en un diccionario, enciclopedia..."

Memoria: "memorizo los apuntes para el día del examen"

Examen: "cambio mi forma de estudiar si el examen es de test o de desarrollo"

Preguntas: "cuando estudio para un examen me planteo preguntas que pueden incluirse en el examen" 


\section{ESTUDIO SOBRE LAS ESTRATEGIAS DE APRENDIZAJE Y EL AUTOCONCEPTO EN ALUMNOS DE ESO Y BACHILLERATO}

Estrategias de estudio y sexo: nos encontramos diferencias significativas $(p<, 01)$ en "amplio información con bibliografía complementaria" $(-, 133)$ con una correlación baja, en "subrayo el material de estudio" (,226) con una correlación baja, en "hago esquemas del material a estudiar" $(, 167)$, con una correlación baja y en "realizo un resumen de los temas a estudiar" $(, 147)$ con una correlación baja. También nos encontramos diferencias significativas $(p<, 05)$ en "antes de estudiar en profundidad suelo hacer una lectura superficial” $(, 102)$ con una correlación baja.

Estrategias de estudio y edad: nos encontramos diferencias significativas $(p<, 01)$ en "cuando estudio relaciono los contenidos de la materia con otras asignaturas" $(, 128)$ con una correlación baja, "subrayo el material de estudio" $(, 139)$ con una correlación baja, "en la realización de esquemas utilizo muchas palabras" $(, 147)$ con una correlación baja y "cambio mi forma de estudiar si el examen es de test o de desarrollo" $(, 137)$ con una correlación baja. También nos encontramos diferencias significativas $(\mathrm{p}<, 05)$ en "realizo un resumen de los temas a estudiar" $(, 113)$ con una correlación baja y "Ios temas que no entiendo los consulto en un diccionario, enciclopedia..." (-,126) con una correlación baja.

Estrategias de estudio y curso: nos encontramos diferencias significativas $(p<.01)$ en "cuando estudio relaciono los contenidos de la materia con otras asignaturas" $(, 160)$ con una correlación baja, "antes de estudiar en profundidad suelo hacer una lectura superficial" (,178) con una correlación baja, "subrayo el material de estudio" $(, 160)$ con una correlación baja, "en la realización de esquemas utilizo muchas palabras" (,175) con una correlación baja y "cambio mi forma de estudiar si el examen es de test o de desarrollo" (,206) con una correlación baja. También encontramos diferencias significativas $(\mathrm{p}<, 05)$ en "realizo un resumen de los temas a estudiar" $(, 125)$ con una correlación baja y "Ios temas que no entiendo los consulto en un diccionario, enciclopedia..." (-,103) con una correlación baja.

Tabla 2. Correlación entre estrategias de aprendizaje y sexo, edad y curso (Parte I)

\begin{tabular}{lrrrrrrrr}
\hline & Relación & Biblio & Materiales & Lectura & Subrayar & Esquema & Palabra \\
\hline Sexo &,- 009 &,$- 133^{* *}$ &, 025 &, $102^{*}$ &, $226^{* *}$ &, $167^{* *}$ &, 039 \\
Edad &, $128^{* *}$ &,- 010 &,- 082 &, 082 &, $139^{* *}$ &,- 064 &, $147^{* *}$ \\
Curso &, $160^{* *}$ &,- 020 &, 022 &, $178^{* *}$ &, $160^{* *}$ &,- 032 &, $175^{* *}$ \\
\hline$* * \mathrm{p}<.01$ & $* \mathrm{p}<.05$ & & & & & & &
\end{tabular}

Tabla 3. Correlación entre estrategias de aprendizaje y sexo, edad y curso (Parte II)

\begin{tabular}{|c|c|c|c|c|c|c|}
\hline & Resumen & Mapas & Diccionario & Memoria & Examen & Preguntas \\
\hline Sexo &, $147^{* *}$ &, 047 &,- 057 & ,086 &,- 015 & ,086 \\
\hline Edad &, $113^{*}$ &,- 077 &,$- 126^{*}$ &,- 012 &, $137^{* *}$ &,- 068 \\
\hline Curso &, $125^{*}$ &,- 076 &,$- 103^{*}$ &,- 011 &, $206^{* *}$ & 070 \\
\hline
\end{tabular}

\section{DISCUSIÓN Y CONCLUSIONES}

En el presenta trabajo se propuso explorar las estrategias de aprendizaje y autoconcepto de alumnos de ESO y Bachillerato.

Las estrategias de aprendizaje son un pilar fundamental en el desarrollo educativo de los alumnos pues sin unas buenas estrategias puede conllevar a un inadecuado aprendizaje y rendimiento 
académico (Aguilar, 2010; Costa y Tabernero, 2012). A su vez el uso de diferentes estrategias de aprendizaje está relacionado con características como la edad, el sexo 0 el curso de los estudiantes Gázquez, Pérez, Ruiz, Miras y Vicente, 2006).

Por otro lado, el autoconcepto, seria otro de los pilares fundamentales en el desarrollo de los estudiantes tanto a nivel educativo como a nivel personal, pues sin él el resto de piezas del engranaje no funciona (Amezcua y Pichardo, 2000; Garaigordobil et al., 2008)

En las limitaciones de este estudio nos encontramos que el cuestionario de estrategias de aprendizaje no está validado en población española.

En futuras investigaciones, se intentaría validar el cuestionario en población española, realizar tablas de contingencia para ver cuáles eran las diferencias entre variables que han correlacionado en el estudio además de poder evaluar también aspectos como la personalidad en los estudiantes y su modificación a lo largo de toda la etapa educativa. En general se necesita más investigación en este campo.

\section{REFERENCIAS}

Aguilar, M. C. (2010). Estilos y estrategias de aprendizaje en jóvenes ingresantes a la universidad. Revista de Psicología, 28, 207-225.

Amezcua, J.A. y Pichardo, C. (2000). Diferencias de género en autoconcepto en sujetos adolescentes. Anales de Psicología, 16, 207-214.

Costa, S. y Tabernero, C. (2012). Rendimiento académico y autoconcepto en estudiantes de Educación Secundaria Obligatoria según el género. Revista Iberoamericana de Psicología y Salud, 3, 175-193.

Fernández, M. P., Martínez, R. y Beltrán, J. A. (2001). Efectos de un programa de entrenamiento en estrategias de aprendizaje. Revista española de pedagogía, 59, 229-250.

Garaigordobil, M., Pérez, J. L., y Mozaz, M. (2008). Self-concept, self-esteem and psychopathological symptoms. Psycothema, 20, 114-123.

Gargallo, B., Suárez, J. y Ferreras, A. (2007). Estrategias de aprendizaje y rendimiento académico en estudiantes universitarios. Revista de Investigación Educativa, 25, 421-441.

Gázquez, J.J., Pérez, M.C., Ruiz, M. I., Miras, F. y Vicente, F. (2006). Estrategias de aprendizaje en estudiantes de enseñanza secundaria obligatoria y su relación con la autoestima. International Journal of Psychology and Psychlogical Therapy, 6, 51-62.

Herrera-Torres, L. y Lorenzo-Quíles, 0. (2009). Estrategias de aprendizaje en estudiantes universitarios. Un aporte a la construcción del Espacio Europeo de Educación Superior. Educación y Educadores, 12, 75-98.

Infante, L., Morena, L. de la, García, B., Sánchez, A., Hierrezuelo, L. y Muñoz, A. (2002). Un estudio sobre el autoconcepto social en estudiantes de E.S.0.: diferencias de género. Revista Electrónica Interuniversitaria de Formación del Profesorado,5, 1-5.

Inglés, C.J., Pastor, Y., Torregrosa, M.S., Redondo, J. y García-Fernández, J.M. (2009). Diferencias en función del género y el curso académico en dimensiones del autoconcepto: estudio con una muestra de adolescentes españoles. Anuario de Psicología, 40, 271-288.

Membrilla, J.A.A., y Martínez, C. P. (2000). Diferencias de género en autoconcepto en sujetos adolescentes. Anales de Psicología, 16, 207-224.

Monereo, C., Pozo, J.I. y Castelló, M. (2001). La enseñanza de estrategias de aprendizaje en el contexto escolar. Psicología de la educación escolar, 2, 235-258.

Musitu, G., García, F., y Gutiérrez, M. (1994). AFA: autoconcepto forma-A. Madrid: TEA Ediciones.

Padilla, M.T., García, S. y Suárez, M. (2010). Diferencias de género en el autoconcepto general y aca- 
démico en estudiantes de 40 de ESO. Revista de Educación, 352, 495-515.

Valenzuela-Zambrano, B., y López-Justicia, M.D. (2015). Autoconcepto de estudiantes universitarios chilenos con discapacidad. Diferencias en función de género. Revista nacional e internacional de educación inclusiva, 8, 153-170).

Yun, D. (2001). A comparison of gender differences in academic self-concept and motivation between high-ability and average Chinese adolescents. Journal of Secondary Gifted Education, 13, 22-32.

Zamora, R. R., y Núnez, L.A.E. (2017). Trabajo colaborativo y estrategias de aprendizaje en entornos virtuales en jóvenes universitarios. RIDE Revista Iberoamericana para la Investigación y el Desarrollo Educativo, 7, 86-109. 